\title{
Laplacian Integral of Particular Steiner System
}

\author{
Alfi Yusrotis Zakiyyah" \\ Mathematics Department, School of Computer Science \\ Bina Nusantara University, \\ Jakarta, Indonesia 11480 \\ alfi.zakiyyah@binus.edu \\ *Correspondence: alfi.zakiyyah@binus.edu
}

\begin{abstract}
The notion of a hypergraph is motivated by a graph. In graph, every edge contains of two vertices. However, a hypergraph edges contains more than two vertices. In this article use hyperedge to mention edge of hypergraph. A linear intersecting hypergraph associate with a finite projective plane of order $n$. This research find that finite projective plane order is Laplacian integral.
\end{abstract}

Keywords: Graph; hypergraph; Laplacian integral; projective plane.

\section{INTRODUCTION}

Let denotes a hypergraph with and are vertex set and hyperedge set respectively. The hypergraph whose all vertices have the same degree is called by -regular. A runiform hypergraph in which all hyperedges have the same cardinality (Rodriguez, 2013).

This research focuses on the Steiner system that is associated with the k-uniform hypergraph. Let $\mathrm{S}(\mathrm{t}, \mathrm{k}, \mathrm{n})$ denotes the Steiner system which satisfied satisfied be integers. Steiner triple systems $\mathrm{S}(2,3, \mathrm{n})$ is one type of Steiner system with $\mathrm{k}=3$ and consider as a 3 -uniform hypergraph. An example of this type is the Fano plane that has seven vertices (Bretto, 2003).

The application of Steiner system concept is applied in balancing storage systems (Chee, et al. 2020). The storage units easily to recover, fast read and write by distributing storage systems to declustered-parity RAIDs by employ dense partial Steiner systems

Another research in the Steiner system about its properties that considered in algebraic geometry/ commutative algebra perspective. (Ballico, et al. 2020) research about how to define Steiner configuration points and their complement that associated with two ideals, in a suitable polynomial ring.

studied about algebraic geometry/commutative algebra in study of Steiner system. They associated that two ideals, in a suitable polynomial ring, defining a Steiner configuration of points and its complement. (Braun et.al, 2016) also studied about q- analog Steiner system properties.

In this study focus on the properties of Laplacian matrix of Steiner system. A Laplacian matrix is a symmetric matrix and positive semidefinite so that all eigenvalues are real and nonnegative. The notion of Laplacian integral hypergraphs are motivated by Laplacian integral graphs. In the study of Laplacian spectrum of graph, (Fallat, et al. 2005) found that the characterization of graph which have distinct integer eigenvalues. In the study about Laplacian integral, Zakiyyah et al. Laplacian integral of complete tripartite hypergraphs . Complete tripartite hypergraphs is Laplacian integral with sufficient condition Another result that joins and products of Laplacian integral graphs are Laplacian integral (Zakiyyah, et al. 2017).

\section{METHODS}

This research study about matrix analysis of finite projective plane. First step, define the Laplacian matrix of this hypergraph. Further step, determine the spectrum hence from the spectrum shown all the the eigenvalues. If all the eigenvalues are integer, finite projective plane can be considered as Laplacian integral hypergraph. 


\section{RESULTS AND DISCUSSION}

There are several type of Steiner system such as finite projective plane, affine plane, Steiner quadruple system Steiner quintuple system, and Steiner triple systems. This research focus on how to determine Laplacian integral finite projective plane.

Let $\mathcal{P}_{\mathrm{n}}$ denote finite projective plane of order $n$. Define that $\mathcal{P}_{\mathrm{n}}$ is a linear intersecting hypergraph satisfy :

1. each vertex consist $n+1$ hyperedges and each hyperedge consist $n+1$ vertices.

2. any two distinct vertices are contained in a unique hyperedges.

3. there exists a set of four vertices such that no three lie on a common hyperedges.

4. each number of hyperedge and vertices are $\mathrm{n}^{2}+\mathrm{n}+1$ respectively

The $n$ partite intersecting hypergraph can be determined by remove a point from a projective plane of order $n$, and all $n+1$ lines through that point hence sides corresponding to the lines that were removed, and the vertex cover number of this hypergraph is $n$.

The example of finite projective plane of order 2 is Fano Plane and called by Fano hypergraph. Fano Plane consist 7 vertices with 3 vertex on every hyperedges and 3 lines through every vertex. For Fano hypergraph let the set of vertices is $V=\{1,2,3,4,5,6\}$ hence the set of hyperedges is $E=\{124,156,137,235,457,346,267\}$.

In this part, we discuss the Laplacian matrix. Let $\mathrm{H}=(\mathrm{V}(\mathrm{H}), \mathrm{E}(\mathrm{H}))$ denotes a hypergraph. There are several representation matrices of hypergraph such as an adjacency matrix, Laplacian matrix, incidence matrix, etc. Let $\mathrm{M}=\mathrm{M}(\mathrm{H})=\left(\mathrm{m}_{\mathrm{ij}}\right)$ and Let $\mathrm{L}=\mathrm{L}(\mathrm{H})=\left(\mathrm{l}_{\mathrm{ij}}\right)$ denote adjacency matrix and Laplacian matrix respectively. Let $\mathrm{D}(\mathrm{H})$ denote diagonal matrix the main diagonal consist of the Laplacian degree of a vertex. According to (Rodriguez, 2003), Laplacian degree of a vertex $v_{i} \in V(H)$ satisfy :

$$
\delta_{l}\left(v_{i}\right)=\sum_{j=1}^{n} m_{i j}
$$

The following theorem states the spectrum of finite projective plane $\mathcal{P}_{\mathrm{n}}$ denoted by $\operatorname{Spec}_{\mathrm{L}}\left(\mathcal{P}_{\mathrm{n}}\right)$ with the first row represent the eigenvalues and its multiplicity in second row

Theorem. Let $\mathcal{P}_{\mathbf{n}}$ denote finite projective plane order $n$, the spectrum of $\mathcal{P}_{\mathbf{n}}$ satisfy.

$$
\operatorname{Spec}_{\mathrm{L}}\left(\mathcal{P}_{\mathrm{n}}\right)=\left(\begin{array}{cc}
0 & n^{2}+n+1 \\
1 & n^{2}+n
\end{array}\right)
$$

Proof. Consider the properties of that any two distinct vertices are contained in a unique hyperedge hence for all entry of adjacency matrix is 1 unless in the diagonal is zero. Also from these properties, we get Laplacian degree $\delta_{1}\left(\mathrm{v}_{\mathrm{i}}\right)=n^{2}+n$.

The Laplacian matrix of $\mathcal{P}_{\mathrm{n}}$ satisfying $\mathrm{L}\left(\mathcal{P}_{\mathrm{n}}\right)=D-M$ the size all of this matrix is $\mathrm{n}^{2}+\mathrm{n}+1$. Let Matrix $D$ is diagonal matrix which all the entries are $\delta_{\mathrm{l}}\left(\mathrm{v}_{\mathrm{i}}\right)$ hence $D=\delta_{\mathrm{l}}\left(\mathrm{v}_{\mathrm{i}}\right) I$ with $I$. Suppose with $\mathrm{M}=J-I$ with $\mathrm{J}$ is ones matrix. Further $\mathrm{L}\left(\mathcal{P}_{\mathrm{n}}\right)=D-M=\left(\delta_{\mathrm{l}}\left(\mathrm{v}_{\mathrm{i}}\right)+1\right) I-J=\left(n^{2}+n+1\right) I-J$

Let $\mathbf{v}$ be any eigenvector of $\mathrm{J}$ with eigenvalue $\mathrm{r}$, where $r$ is either $n^{2}+n+1$ or 0 .

Then we have $\left(\left(n^{2}+n+1\right) I-J\right) \mathbf{v}=\left(n^{2}+n+1\right) \boldsymbol{v}-\mathbf{r v}$

$$
=\left(\left(n^{2}+n+1\right)-r\right)
$$

so $\left(\left(n^{2}+n+1\right)-r\right)$ is an eigenvalue of $\mathrm{L}\left(\mathcal{P}_{\mathrm{n}}\right)$ with eigenvector $\mathrm{v}$. Since $\mathrm{J}$ has a basis of eigenvectors, $\mathrm{n}^{2}+\mathrm{n}+1$ and 0 are all the eigenvalues of $\operatorname{J~}\left(\mathcal{P}_{n}\right)$.

\section{CONCLUSION}

According to the result, we can consider that finite projective plane order $n$ is Laplacian integral. Further research, we search about the application and another properties of representation matrix of finite projective plane order $n$.

\section{REFERENCES}

Bretto, A. (2013). Hypergraph theory: An introduction. Mathematical Engineering. Cham:

Springer.

Ballico, E., Favacchio, G., Guardo, E., \& Milazzo, L. (2020). Steiner systems and

configurations of points. Designs, Codes and Cryptography, 1-21.

Braun, M.,Etzion, T., Ostergard,.P., Vardy, A., and Wassermann,A. (2016). Existence of

$\mathrm{q}$ - analogs of Steiner system . Forum of Mathematics , Pi, 4, E7. doi : 10.1017/fmp.2016.5

Chee, Y. M., Colbourn, C. J., Dau, H., Gabrys, R., Ling, A. C., Lusi, D., \& Milenkovic, O. (2020). Access balancing in storage systems by labeling partial Steiner systems. Designs, Codes and Cryptography, 88(11), 2361-2376.

Fallat, S.M., Kirkland, S.J., Molitierno, J.J. and Neumann, M., 2005. On graphs whose Laplacian matrices have distinct integer eigenvalues. Journal of Graph Theory, 50(2), pp.162-174.

Li, S., Kang, L., Shan, E., \& Dong, Y. (2018). The finite projective plane and the 5-Uniform linear intersecting hypergraphs with domination number four. Graphs and Combinatorics, 34(5), 931-945.

Rodriguez, J. A. (2003). On the Laplacian spectrum and walk-regular hypergraphs. Linear and Multilinear Algebra, 51(3), 285-297

Zakiyyah, A.Y., Garminia, H., Salman, A.N.M. On the characterization of Laplacian integral complete tripartite hypergraphs Far East Journal of Mathematical Sciences 102 (9), 2133-2142 | vol: | issue: | 2017 